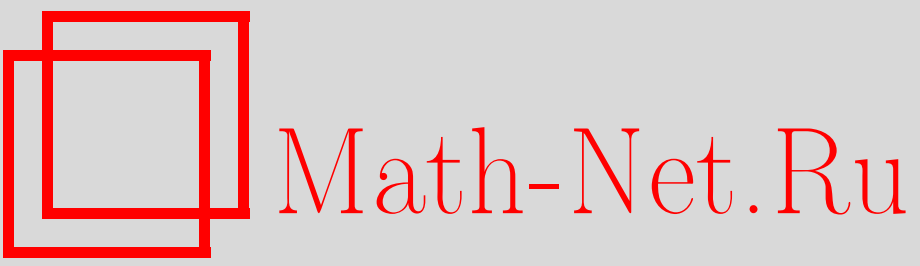

М. А. Ермоленко, А. Ф. Федотов, Численный анализ влияния размеров оболочки на закономерности уплотнения и формоизменения при CBC-прессовании, Вестн. Сам. гос. техн. ун-та. Сер. Физ.-мат. науки, 2002, выпуск 16, 75-80

DOI: https://doi.org/10.14498/vsgtu100

Использование Общероссийского математического портала Math-Net.Ru подразумевает, что вы прочитали и согласны с пользовательским соглашением

http://www.mathnet.ru/rus/agreement

Параметры загрузки:

IP: 35.173 .219 .149

26 апреля 2023 г., 14:47:18 


\title{
ЧИСЛЕННЫЙ АНАЛИЗ ВЛИЯНИЯ РАЗМЕРОВ ОБОЛОЧКИ НА ЗАКОНОМЕРНОСТИ УПЛОТНЕНИЯ И ФОРМОИЗМЕНЕНИЯ ПРИ СВС-ПРЕССОВАНИИ
}

\begin{abstract}
Выполнено исследование на сходимость численного решения краевой задачи пластического деформирования и определены размеры сетки конечных элементов для дискретной модели процесса $C B C$ прессования. Установлен экстремальный характер изменения плотности заготовки в зависимости от толщины оболочки, которая определяет ее ресурс сжимаемости. Для получения высокоплотных заготовок необходимо, чтобы ресурс сжимаемости оболочки был выше ресурса сжимаемости заготовки. С уменьшением радиальных размеров оболочки возможно усиление искажения формы заготовки из-за неоднородности напряженно-деформированного состояния вблизи поверхностей инструмента с внешним трением.
\end{abstract}

При прямом способе получения компактных материалов неостывшие продукты самораспространяющегося высокотемпературного синтеза (CBC) подвергают прессованию в закрытой матрице с передачей давления через сыпучую оболочку (технология $\mathrm{CBC}$-прессования). В качестве оболочки используется песок. Оболочка выполняет две функции: осуществляет теплоизоляцию саморазогревающихся продуктов синтеза от холодного инструмента и передает давление на продукты СВС. Размеры оболочки определяют температурный режим деформации, напряженно-деформированное состояние и закономерности уплотнения продуктов синтеза. Анализ влияния размеров оболочки на температурный режим процесса $\mathrm{CBC}$-пресссования выполнен в [1]. Экспериментальных или иных исследований связи размеров оболочки с механикой процесса не проводилось. Цель работы - изучение влияния размеров оболочки на закономерности уплотнения и формоизменения при СВС-прессовании. Наиболее эффективно такое исследование провести на математической модели процесса. Адекватная математическая модель процесса СВС-прессования получена в [2] при решении методом конечных элементов (МКЭ) краевой задачи пластического деформирования в закрытой матрице пористой заготовки из горячих продуктов синтеза и сыпучей оболочки. Эта модель используется в настоящей работе для исследования влияния размеров оболочки на процессы уплотнения и формоизменения при СВС-прессовании.

При численных методах решения к числу основных относится вопрос выбора размеров элементов сетки для дискретной модели объекта. Проблема выбора размеров сетки приобретает особое значение при решении задач с варьированием размеров моделируемого объекта. В этом случае корректное сопоставление результатов исследования возможно при условии инвариантности размеров элементов сетки к размерам объекта. Очевидно, что с увеличением размеров объекта неизбежно растут затраты машинного времени. С целью определения рациональных размеров сетки конечных элементов было проведено исследование на сходимость численного решения краевой задачи пластического деформирования при $\mathrm{CBC}$-прессовании. Для задач, имеющих точное аналитическое решение, сходимость оценивается сопоставлением численного и точного решений. При исследовании сходимости задач, для которых нет точного решения, применяют апостериорную оценку по правилу Рунге [3]. Сначала находят решение при шаге пространственных координат $h$, потом вычисляют решение при шаге $h / 2$ и сравнивают значения $y_{h}\left(x_{i}\right)$ и $y_{h / 2}\left(x_{i}\right)$ в одинаковых узлах:

$$
\delta_{i}=y_{h}\left(x_{i}\right)-y_{h / 2}\left(x_{i}\right),
$$

где $\delta_{i}$ - ошибка решения в $i$-том узле; $x_{i}-$ вектор узловых координат. Затем выполняется проверка точности полученного решения:

$$
\|\delta\| \leq \varepsilon .
$$

Здесь $\|\delta\|$ - принятая норма ошибки решения; $\varepsilon$ - заданная точность решения задачи. Если условие (2) выполняется, то решение $y_{h}\left(x_{i}\right)$ принимается за искомое. В противном случае шаг $h / 2$ делят пополам и вычисляют решение $y_{h / 4}\left(x_{i}\right)$. Контроль точности в вычислении значений $y_{h / 4}\left(x_{i}\right)$ осуществляют аналогично предыдущему. Точного решения краевой задачи пластического деформирования при СВС-прессовании нет и исследование численного решения на сходимость в настоящей работе выполнялось по правилу Рунге.

Рассматривался процесс CBC-прессования круглых заготовок и осесимметричное пластическое течение в цилиндрических координатах $r$ и $z$. При дискретизации на КЭ исследуемая 
область покрывалась прямоугольниками длиной $h_{r}$ по координате $r$ и высотой $h_{z}-$ по координате $z$. Полученные прямоугольники диагональю разбивались на два треугольника. В механике порошков и пористых тел главное внимание уделяется не исследованию напряженнодеформированного состояния, а изучению параметров, характеризующих состояние материала и, в первую очередь, его плотности. При СВС-прессовании в заготовке и оболочке формируется неоднородное поле плотности. Поэтому в качестве решения рассматривалось распределение относительной плотности оболочки $\left(\rho_{2}\right)$ по радиусу $\rho_{2}(r)$ и по высоте $\rho_{2}(z)$ деформируемого объема. Зависимость $\rho_{2}(r)$ определяли в горизонтальном слое, расположенном вдоль верхней опорной плоскости заготовки; зависимость $\rho_{2}(z)$ - в вертикальном столбце, прилегающем к боковой поверхности заготовки. За норму $\|\delta\|$ принималась величина максимальной ошибки решения по ансамблю конечных элементов рассматриваемых слоев оболочки:

$$
\|\delta\|=\max \left|\delta_{i}\right|
$$

Моделировался процесс СВC-прессования круглых заготовок из сплава $\mathrm{TiC}-20 \% \mathrm{Ni}$ радиусом $R_{1}=39$ мм в пресс-форме с матрицей радиусом $R_{\mathrm{M}}=62,5$ мм, давлении прессования $q=120$ МПа и скорости плунжера $v_{0}=8 \mathrm{mм} / \mathrm{c}$. Начальная высота заготовки составляла $h_{10}=14 \mathrm{mм;} \mathrm{об-}$ щая высота - 34 мм при одинаковой толщине верхней и нижней оболочек, равной 10 мм. Начальный размер ячеек сетки принимался равным для заготовки - $h_{r}=4,87$ мм, $h_{z}=3,5$ мм; для оболочки $-h_{r}=4,7$ мм, $h_{z}=5$ мм. Геометрические характеристики дискретных моделей приведены в табл. 1. Точность решения составляла $\varepsilon=0,2 \%$. Расчет выполнялся на ПЭВМ PentiumIV с частотой процессора 1600 МГц. Программирование осуществлялось в среде Visual Basic 6.0.

Т а б л и ц а 1

Геометрические и топологические характеристики дискретных моделей

\begin{tabular}{|c|c|c|c|c|c|c|c|c|c|}
\hline \multirow[t]{2}{*}{ Модель } & Объект & \multicolumn{2}{|c|}{ Заготовка } & \multicolumn{3}{|c|}{ Оболочка } & \multicolumn{2}{|c|}{ Число } & \multirow{2}{*}{$\begin{array}{c}\text { Время } \\
\text { расчета, } \\
\text { ч }\end{array}$} \\
\hline & $r$, мM & $0-35$ & $35-39$ & $39-45$ & $45-54,5$ & $54,5-62,5$ & КЭ & узлов & \\
\hline 1 & $\begin{array}{l}h_{r}, \mathrm{MM} \\
h_{z}, \mathrm{MM}\end{array}$ & \multicolumn{2}{|c|}{$\begin{array}{l}4,87 \\
3,50\end{array}$} & \multicolumn{3}{|c|}{$\begin{array}{l}4,70 \\
5,00\end{array}$} & 208 & 147 & 0,17 \\
\hline 2 & $\begin{array}{l}h_{r}, \mathrm{MM} \\
h_{z}, \mathrm{MM}\end{array}$ & \multicolumn{2}{|c|}{3,25} & \multicolumn{3}{|c|}{$\begin{array}{l}3,36 \\
3,33\end{array}$} & 456 & 291 & 1,1 \\
\hline 3 & $\begin{array}{l}h_{r}, \mathrm{MM} \\
h_{z}, \mathrm{MM}\end{array}$ & \multicolumn{2}{|c|}{1,75} & \multicolumn{3}{|c|}{2,6} & 800 & 483 & 7,0 \\
\hline 4 & $h_{r}, \mathrm{MM}$ & 3,5 & 1,33 & 1,5 & 4,75 & 2,0 & 920 & 541 & 12,3 \\
\hline & $h_{z}, \mathrm{MM}$ & \multicolumn{2}{|c|}{1,4} & \multicolumn{3}{|c|}{2,0} & & & \\
\hline \multirow[t]{2}{*}{5} & $h_{r}, \mathrm{MM}$ & 3,5 & 1,0 & 1,0 & 4,75 & 2,0 & 720 & 436 & 5,0 \\
\hline & $h_{z}, \mathrm{MM}$ & \multicolumn{2}{|c|}{2,0} & \multicolumn{3}{|c|}{2,5} & & & \\
\hline \multirow[b]{2}{*}{6} & $h_{r}, \mathrm{MM}$ & 3,5 & 1,33 & 1,5 & 4,75 & 2,0 & & & \\
\hline & $h_{z}, \mathrm{MM}$ & \multicolumn{2}{|c|}{2,33} & \multicolumn{3}{|c|}{$\begin{array}{l}2,5 \text { при } h_{2 \mathrm{H}} \leq 10 \mathrm{мм} \\
5,0 \text { при } h_{2 \mathrm{~B}} \geq 15 \mathrm{Mм}\end{array}$} & 644 & 393 & 3,25 \\
\hline
\end{tabular}

Характер изменения зависимостей $\rho_{2}(r)$ и $\rho_{2}(z)$ при измельчении сетки КЭ представлен на рис. 1 и 2. При неоднородном распределении плотности в зонах с малыми градиентами сходимость достигается на более крупных ячейках по сравнению с зонами высоких градиентов плотности (см. рис. 1). Поэтому после получения сходимости в области малых градиентов на следующем шаге измельчения сетки выполняли только в области высоких градиентов плотности. Окончательные размеры неравномерной сетки, обеспечивающие заданную точность решения, соответствуют дискретной модели 6 (табл. 1). При толщине нижней или верхней оболочек свыше 15 мм сходимость обеспечивается при шаге сетки $\left(h_{z}\right)$ в оболочке, равном $h_{z}=5$ мм. Вычислительный алгоритм рассматриваемой краевой задачи содержит два вложенных итерационных цикла - внутренний при решении физически нелинейной задачи пластического течения методом гидродинамических приближений и внешний при расчете сил внешнего

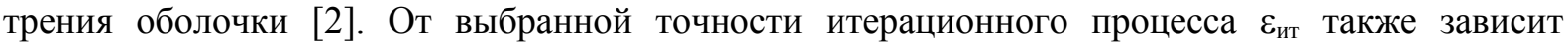
сходимость численного решения. Численным экспериментом установлено, что сходимость обеспечивается при $\varepsilon_{\text {ит }}=2 \%$ для обоих итерационных циклов. Заданная точность решения задачи пластического течения достигалась за счет автоматического выбора шага по времени нагружения $\Delta t$. При этом максимальное число итераций в методе гидродинамических приближений для текущей величины шага $\Delta t$ принималось равным трем. Если за 3 итерации 76 
принималось равным трем. Если за 3 итерации процесс не сходился, то время $\Delta t$ уменьшалось вдвое, и расчет повторялся. Начальный шаг по времени составлял $\Delta t=0,05$ с.

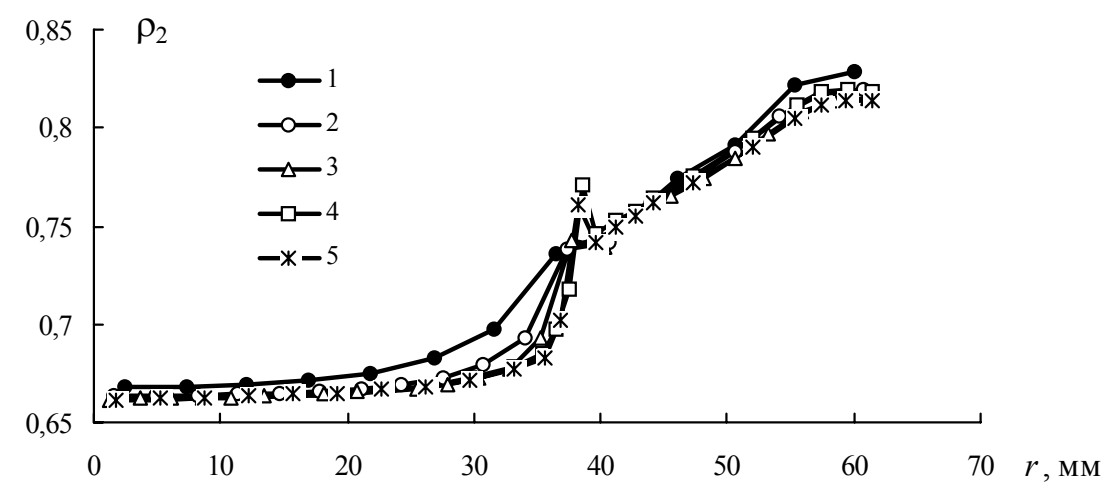

Р и с. 1. Влияние размеров сетки на распределение плотности оболочки по радиусу матрицы $\rho_{2}(r)$ (обозначения - в табл. 1)

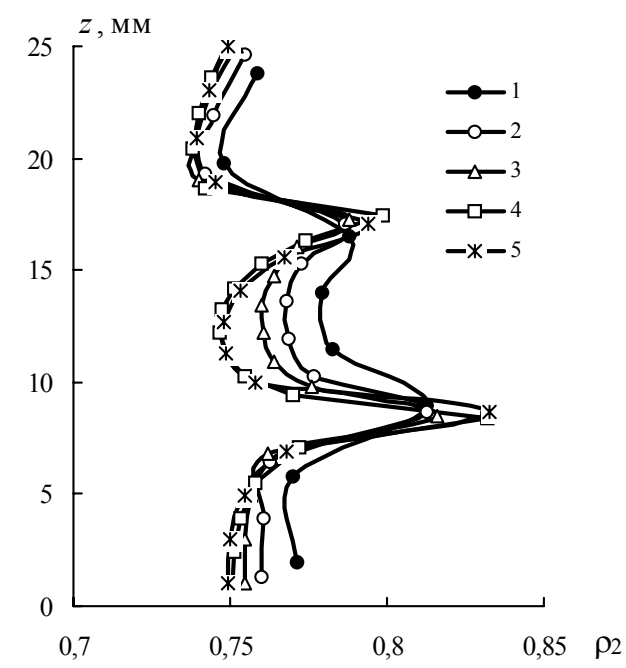

Р и с. 2. Влияние размеров сетки на распределение плотности оболочки по высоте матрицы $\rho_{2}(z)$ (обозначения - в табл. 1)

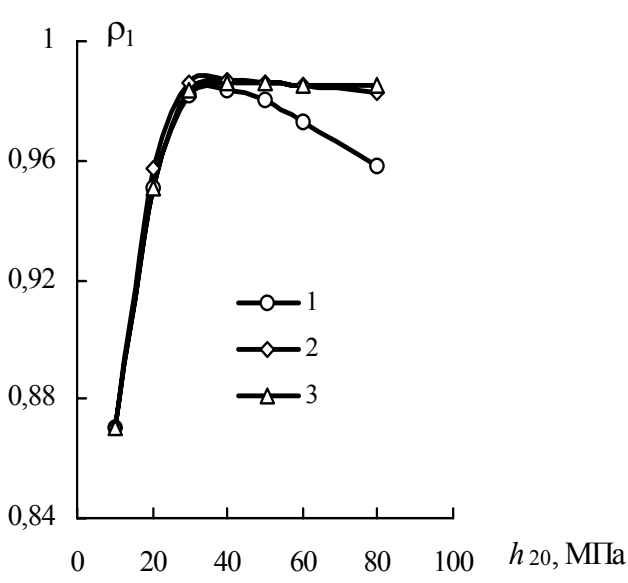

Р и с. 3. Влияние толщины оболочки на плотность заготовки при ее расположении в верхней (1), средней (2) и нижней

(3) части матрицы

Исследование влияния толщины оболочки на закономерности уплотнения заготовки проводилось на шагах с пространственными координатами $h_{r}$ и $h_{z}$ для дискретной модели 6 (см. табл. 1). Начальная толщина оболочки $h_{20}$ включает толщину нижней $h_{2 \text { н }}$ и верхней $h_{2 \text { в }}$ оболочек: $h_{20}=h_{2 \mathrm{H}}+h_{2 \mathrm{~B}}$. Рассматривалось три варианта расположения заготовки в оболочке. В 1-м варианте заготовка размещалась в центральной части матрицы и одновременно изменялась толщина верхней, и нижней оболочек. Во 2-м варианте заготовка находилась в нижней части матрицы. При этом толщина нижней оболочки оставалась постоянной $\left(h_{2 \mathrm{H}}=5\right.$ мм), а варьировалась только толщина верхней оболочки. В 3-м варианте заготовка располагалась в верхней части матрицы. В этом случае фиксировалась толщина верхней оболочки ( $h_{2 \mathrm{~B}}=5$ мм), а изменялась только толщина нижней оболочки.

На рис. 3 приведены зависимости средней относительной плотности заготовки $\rho_{1}$ от начальной толщины $h_{20}$ оболочки для трех вариантов расположения заготовки в матрице. Согласно расчетам независимо от положения в матрице максимальная плотность заготовки $\rho_{1}$ обеспечивается при начальной толщине оболочки $h_{20}=35$ - 40 мм. Следует отметить резкое уменьшение плотности прессованной заготовки при $h_{20}<30$ мм. Для объяснения этого явления рассмотрим процесс совместного деформирования и уплотнения заготовки и оболочки. 
Из рис. 1 следует, что в наибольшей степени уплотняются объемы оболочки, расположенные в кольцевой зоне вокруг заготовки. В меньшей степени уплотняется оболочка центральной зоны, в которой находится заготовка. Уплотнение заготовки и оболочки обусловлено, главным образом, осевой деформацией, которая на порядок выше, чем радиальная деформация [2]. Если пренебречь радиальной деформацией, то для качественного анализа процесса деформирования достаточно рассмотреть одномерную механическую модель. Учитывая характер распределения плотности в радиальном направлении (см. рис. 1), эта модель представляет собой два параллельно соединенных элемента - центральная зона с заготовкой и кольцевая зона. Пусть заготовка имеет начальную относительную плотность $\rho_{10}$ и начальную высоту $h_{10}$. Тогда для уплотнения до беспористого состояния с $\rho_{1}=1$ заготовку необходимо осадить на величину

$$
\Delta h_{1}=h_{10}\left(1-\rho_{10}\right) \text {. }
$$

Совместно с осадкой заготовки происходит осадка кольцевой зоны оболочки. Максимальная осадка кольцевой оболочки по аналогии с (4) составляет

$$
\Delta h_{2}=h_{0}\left(1-\rho_{20}\right),
$$

где $h_{0}$ - начальная высота всего деформируемого объема: $h_{0}=h_{10}+h_{20}$. Конечная плотность заготовки будет определяться соотношением начальной плотности и высоты заготовки, а также кольцевой оболочки. Рассмотрим предельный случай, когда заготовка и оболочка имеют одинаковую начальную высоту: $h_{10}=h_{0}$. Если начальная плотность заготовки выше, чем у оболочки $\left(\rho_{10}>\rho_{20}\right)$, то получим $\Delta h_{1}<\Delta h_{2}$ и теоретически возможно получение беспористой заготовки. Напротив, при более высокой начальной плотности оболочки $\left(\rho_{10}<\rho_{20}\right)$ имеем $\Delta h_{1}>\Delta h_{2}$ и сначала до беспористого несжимаемого состояния уплотняется кольцевая оболочка, а заготовка остается пористой. Именно этот случай имеет место при СВС-прессовании сплава $\mathrm{TiC}-20 \%$ $\mathrm{Ni}$ в песчаной оболочке. Начальная относительная плотность продуктов синтеза составляет $\rho_{10}$ $=0,5$; песка $-\rho_{20}=0,61$. В рамках одномерной модели для получения беспористой заготовки необходима осадка на половину ее начальной высоты: $\Delta h_{1}=0,5 h_{10}$. Но кольцевая оболочка уже после осадки на $39 \%$ исчерпывает запас сжимаемости. С увеличением начальной высоты оболочки $h_{20}$ повышается ресурс сжимаемости кольцевой зоны $\Delta h_{2}$, что в итоге приводит к повышению плотности прессуемой заготовки. Таким образом, для получения методом СВСпрессования высокоплотных или беспористых заготовок необходимо обеспечить определенный ресурс сжимаемости оболочки, который характеризуется толщиной оболочки. Минимально допустимая толщина оболочки $h_{2 \min }$ зависит от начальной высоты заготовки и начальной плотности уплотняемых материалов. Для рассматриваемого процесса расчетная величина этого параметра составляет $h_{2 \min }=35-40 \mathrm{Mм}$.

На характер зависимости $\rho_{1}\left(h_{20}\right)$ при $h_{20}>40$ мм оказывает влияние положение заготовки в матрице. При расположении заготовки в верхней или средней части матрицы увеличение $h_{20}$ свыше 40 мм практически не приводит к изменению плотности заготовки. Если заготовка находится в нижней части матрицы, то с увеличением толщины оболочки плотность заготовки уменьшается. В этом случае проявляется влияние сил внешнего трения оболочки. Известно, что из-за внешнего трения порошкового материала давление прессования уменьшается от верхней к донной части матрицы. Для малой толщины оболочки $\left(h_{2}<40\right.$ мм) перепад давления по высоте незначителен и конечная плотность заготовки не зависит от ее положения в матрице. При толщине оболочки свыше 40 мм давление в нижней части становится заметно меньше, чем в средней и особенно в верхней части матрицы, и плотность за-

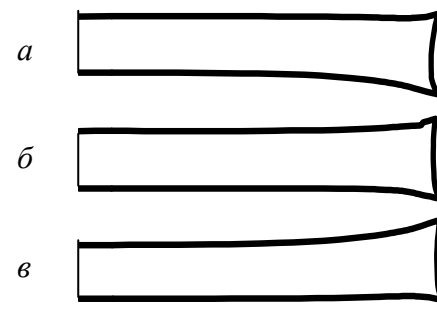

$\Delta h_{\text {заг }}=3,19 \mathrm{MM}$

$\Delta h_{\text {заг }}=2,69 \mathrm{MM}$

$\Delta h_{\text {заг }}=2,94 \mathrm{MM}$

Р и с. 4. Форма сечения заготовки при ее расположении в верхней (1), средней (2) и нижней (3) частях матрицы готовки снижается.

Плотность заготовки - не единственный показатель качества процесса СВС-прессования. Важной технической характеристикой является размерная точность заготовки, которая определяет трудоемкость последующих операций механической обработки. Различие реологических свойств продуктов СВС и песка приводит к искажению формы заготовки - высота ее центральной части меньше, чем периферийной и опорные поверхности являются вогнутыми [2]. Количественной мерой размерной точности может слу- 


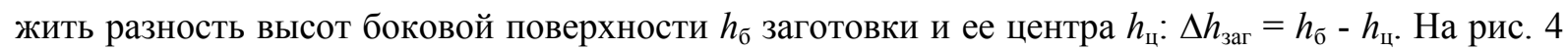
показана форма радиального сечения заготовки в зависимости от ее положения в матрице с радиусом $R_{\mathrm{M}}=62,5$ мм. При расположении заготовки в верхней или нижней части матрицы максимальное искажение имеют опорные поверхности со стороны оболочки с малой толщиной соответственно верхняя (рис. 4а) или нижняя (рис. 4в). При расположении заготовки в центральной части матрицы опорные поверхности искажаются в равной степени (рис. 4б). Для этого варианта имеет место минимальное искажение формы заготовки $\Delta h_{\text {заг }}$.

Влияние радиуса матрицы $\boldsymbol{R}_{\mathrm{M}}$ на среднюю плотность заготовки

Т а б л и ц а 2

\begin{tabular}{|c|c|c|c|c|c|}
\hline$R_{\mathrm{M}}$ мм & 62,5 & 55 & \multicolumn{2}{|c|}{50} & 45 \\
\hline$q$, Мпа & 120 & 156 & 188 & 233 & 233 \\
$\rho_{1}$ & 0,986 & 0,99 & 0,992 & 0,994 & 0,995 \\
\hline
\end{tabular}

Наряду с анализом влияния осевых размеров (толщины $h_{20}$ ) оболочки проведено исследование влияния радиальных размеров оболочки (радиуса матрицы $R_{\mathrm{m}}$ ) на закономерности уплотнения и формоизменения заготовки. Выбор радиальных размеров матрицы имеет принципиальное значение, так как усилие пресса ограничено по величине и именно радиус матрицы определяет рабочее давление прессования. В табл. 2 приведены результаты численного анализа влияния радиуса матрицы $R_{\mathrm{M}}$ на плотность прессованных заготовок при постоянном усилии прессования, равном $Q_{\kappa}=1480$ кН. Исследование проводилось при толщине оболочки $h_{20}=40$ мм и расположении заготовки в средней части матрицы. С уменьшением радиуса матрицы $R_{\text {м }}$ при $Q_{\kappa}=$ const давление прессования $q$ увеличивается и средняя плотность заготовки $\rho_{1}$ возрастает (табл. 2).

С точки зрения получения высокоплотных заготовок естественна ориентация на использование малогабаритных пресс-форм и высоких давлений прессования. Вместе с тем не известно как происходит формоизменение заготовок с изменением радиальных размеров оболочки. Из-

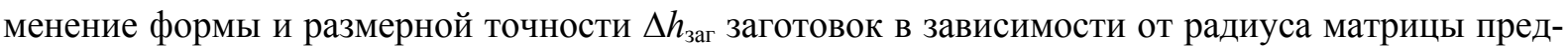
ставлено на рис. 5 . Минимальное значение $\Delta h_{\text {заг }}$ соответствует прессованию в матрице с радиусом $R_{\mathrm{M}}=45$ мм. Однако при этом происходит существенное искривление самих опорных поверхностей (рис. 5д). Как уже отмечалось, с уменьшением радиуса матрицы растет давление прессования. Поэтому снижение размерной точности заготовок при $R_{\mathrm{M}}=45$ мм может быть связано либо с геометрическим фактором (радиус $R_{\text {м}}$ ), либо силовым фактором (давление $q$ ). Для ответа на этот вопрос был выполнен расчет прессования в матрице радиусом $R_{\mathrm{M}}=50$ мм, но при давлении прессования, которое соответствует прессованию в матрице с $R_{\mathrm{M}}=45$ мм. Результаты расчета показывают, что при одинаковом давлении $q$ искажение формы заготовки при прессовании с $R_{\mathrm{M}}=50$ мм (рис. 5г) существенно меньше, чем при прессовании $R_{\mathrm{M}}=45$ мм (рис. 5 д). Следовательно, на размерную точность заготовки оказывают влияние радиальные размеры инструмента и оболочки. С точки зрения размерной точности оптимальным является прессование в матрице с $R_{\mathrm{M}}=50$ мм (рис. 5 в).

Закономерности уплотнения и изменения формы заготовки обусловлены напряженнодеформированным состоянием, которое при СВС-прессовании является неоднородным. Эта неоднородность вызвана как различием реологических свойств заготовки и оболочки [2], так и внешним трением оболочки. Именно области прессовок вблизи поверхностей (и с трением) характеризуются сильной неоднородностью напряженнодеформированного состояния и поля плотности [4]. Поэтому при уменьшении радиальных размеров оболочки часть заготовки попадает

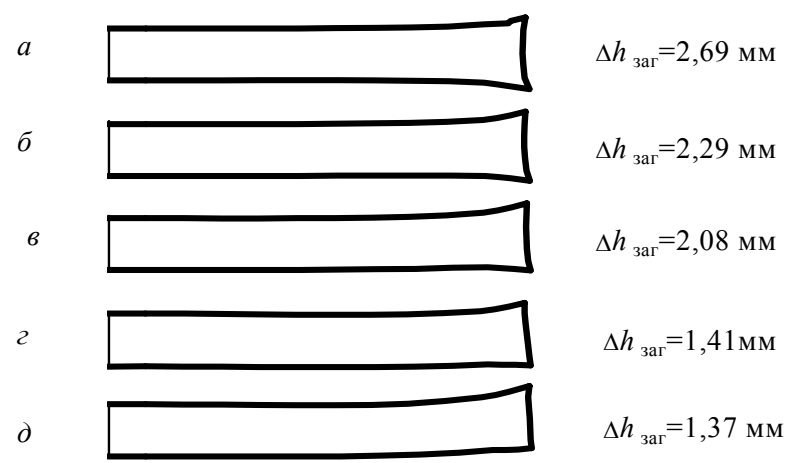

Р и с. 5. Влияние радиуса матрицы на форму заготовки: $a-R_{\mathrm{M}}=62,5 \mathrm{MM} ; \sigma-R_{\mathrm{M}}=55 \mathrm{MM} ; \theta-R_{\mathrm{M}}=50 \mathrm{MM}, q=188 \mathrm{MПа:}$ $2-R_{\mathrm{M}}=50 \mathrm{MM}, q=233 \mathrm{MПа} ; \partial-R_{\mathrm{M}}=45 \mathrm{MM}$ 
в область сильной неоднородности, вызванной силами внешнего трения, и ее размерная точность уменьшается.

Таким образом, выполнено исследование на сходимость численного решения краевой задачи осесимметричного пластического деформирования и определены оптимальные размеры сетки конечныХ элементов для дискретной модели процесса СВС-прессования.

Установлен экстремальный характер изменения плотности заготовки в зависимости от толщины оболочки, которая определяет ее ресурс сжимаемости. Для получения высокоплотных заготовок необходимо, чтобы ресурс сжимаемости оболочки был выше ресурса сжимаемости заготовки.

Размерная точность $\mathrm{CBC}$-прессованной заготовки зависит от ее положения в матрице и радиальных размеров оболочки. С уменьшением радиальных размеров оболочки возможно усиление искажения формы заготовки из-за неоднородности напряженно-деформированного состояния вблизи поверхностей инструмента с внешним трением.

\section{БИБЛИОГРАФИЧЕСКИЙ СПИСОК}

1. Амосов А.П., Федотов А.Ф. Конечно-элементная плоская модель теплового режима при самораспространяющемся высокотемпературном синтезе заготовок в сыпучей оболочке // ИФЖ. 2001. Т. 74. № 5. C. 160-166.

2. Федотов А.Ф., Ермоленко М.А. Конечно-элементная модель процесса осесимметричного пластического деформирования при СВС-прессовании // Вестник СамГТУ. Сер. Физ.- мат. науки. 2001. Вып. 12. C. 91-103.

3. Крылов В.И., Бобков В.В., Монастырный П.И. Вычислительные методы. Т. 2. М.: Наука. 1977. 399 с.

4. К Кипарисов С.С., Либенсон Г.А. Порошковая металлургия. М.: Металлургия, 1991. 432 с. 